\title{
Comparison of clinical efficacy between decitabine combined with half the amount of CAG regimen with CAG regimen alone in patients with inermediate to high-risk myelodysplastic syndrome
}

\author{
Zhenwei $\mathrm{Fan}^{1, \mathrm{a}}$, Xuan Wang ${ }^{2}$, Chunshan Zhao ${ }^{1}$, Wei Wang ${ }^{2}$ and Tingting $\mathrm{Yu}^{2}$ \\ ${ }^{1}$ College Nursing of Beihua University, 132000, jilin city, jilin province, China \\ ${ }^{2}$ Hematology of Affiliated Hospital of Beihua University, 132000, jilin city, jilin province, China
}

\begin{abstract}
Objective: To investigate the efficacy and safety of decitabine combined with half the amount of CAG and CAG regimen alone. Methods: Comparison the effectiveness, overall survival and incidence of adverse reactions of 42 cases of MDS used decitabine combined with half the amount of CAG regimen (decitabine $20 \mathrm{mg} / \mathrm{m}^{2}$, once a day, d1-3, Accra neomycin $7 \mathrm{mg} / \mathrm{m}^{2}$,intravenous injection, once a day, $\mathrm{d} 4-7$, cytarabine $10 \mathrm{mg} / \mathrm{m}^{2}$, every 12 hours, $\mathrm{d} 4-10$, granulocyte colony stimulating factor $300 \mu \mathrm{g}$, once a day, d4-10, white blood cell count $>20 \times 10^{9} / \mathrm{L}$ when deactivated for four courses) and 48 patients in MDS were treated with chemotherapy alone CAG (Accra neomycin $14 \mathrm{mg} / \mathrm{m}^{2}$,once a day, $\mathrm{d} 1-3$, cytarabine $10 \mathrm{mg} / \mathrm{m}^{2}$, every 12 hours, $\mathrm{d} 1-14$, granulocyte colony stimulating factor $300 \mu \mathrm{g}$, once a day, d1-14, white blood cell count $>20 \times 10^{9} / \mathrm{L}$ when deactivated). Results: Compared with pure CAG, clinical efficacy of decitabine combined with half the amount of CAG regimen is better, the treatment does not increase the risk.
\end{abstract}

\section{Introduction}

Myelodysplastic syndrome (MDS) are a group disease, begins at hematopoietic stem cells, In blood cell dysplasia of the main features, there may be abnormal of refractory blood cytoplasm and the amount, prone to transformation of acute myeloid leukemia. Since the majority of patients with the disease are elderly, poor physical function, and the ways of low disease malignant clonal proliferation, bone marrow dysplasia, lead to the treatment of drug sensitivity and the poor resistance, so the prognosis is poor. With the progress of research on epigenetics, DNA methyltransferase inhibitorto,in decitabine represented, make MDS patients get better control and mitigation in clinical application manipulation, especially in patients with RAEB or have been converted into leukemia. In this study, in order to find a more rational and effective treatment, survival, the remission rate and incidence of adverse reactions were compared between two groups patients, who suffer high-risk MDS, the application of decitabine joint half the amount of CAG and simple application of CAG regimen.

\footnotetext{
${ }^{a}$ Corresponding author: Zhenwei Fan: 22289671@qq.com
} 


\section{Materials and method}

\subsection{Object}

Observation group patients have been 31patients with MDS treated in the Hematology,from January 2010 to December 2014, in Affiliated Hospital of North China University. Inclusion criteria: All patients underwent bone marrow puncture, press MICM classification inspection, are diagnostic for MDS in RAEB-1 and RAEB-2 in line with 2008 WHO revised classification criteria. Of which 14 males and 17 females; median age is 61 (41-70) years; RAEB-1 13 cases, RAEB-2 18 cases; treatment median absolute neutrophil before $0.73(0.21-1.81) \times 10^{9} / \mathrm{L}$; median hemoglobin value of $72(51-88)$ $\mathrm{g} / \mathrm{L}$; median platelet count is $28(3-64) \times 10^{9} / \mathrm{L}$; median proportion of bone marrow blasts is 13 (6$18) \%$; karyotype groups: good 10 groups, the middle 13 groups, poor 8 groups; IPSS risk groups: intermediate risk I 9 cases, the intermediate risk II 15 cases, high-risk 7 cases, agreed after communicating with the family application decitabine combined with half the amount of CAG regimen.

Patients in the control group have been 37 patients with MDS treated in the Hematology,from January 2008 to December 2014, in the Affiliated Hospital of North China University hospitalized. Inclusion criteria with the former, of which 18 males and 19 females; median age 57 (36-77) years; RAEB-1 16 Li, RAEB-2 21 cases; treatment median absolute neutrophil before $0.62(0.18-1.97) \times$ $10^{9} / \mathrm{L}$; median hemoglobin value of $73(48-92) \mathrm{g} / \mathrm{L}$; median platelet count of $22(4-73) \times 10^{9} / \mathrm{L}$; median proportion of bone marrow blasts 11 (6-17) \%; karyotype groups: good 12 groups, 16 groups of intermediate, poor 9 groups; IPSS risk groups: patients with intermediate risk I 12, intermediate risk II 17 cases, 8 cases of high risk, all the families of patients have agreed with CAG regimen.

Two groups of data,for statistical analysis, patient's age, sex, the median white cell count, the median hemoglobin values, the median platelet values karyotype groups, RAEB-1 and RAEB-2 are two $\mathrm{P}>0.05$, the difference was not statistically significant.

\subsection{Treatment}

Decitabine joint half the amount of CAG: decitabine $20 \mathrm{mg} / \mathrm{m}^{2}$, once a day,d1-3, Accra neomycin $7 \mathrm{mg} / \mathrm{m}^{2}$, intravenous injection, once a day, $\mathrm{d} 4-7$, cytarabine $10 \mathrm{mg} / \mathrm{m}^{2}$, every 12 hours, $\mathrm{d} 4-10$, granulocyte colony stimulating factor $300 \mu \mathrm{g}$, once a day, $\mathrm{d} 4-10$, white blood cell count $>20 \times 10^{9} / \mathrm{L}$ when deactivated for four courses.

CAG simple scheme: Accra neomycin $14 \mathrm{mg} / \mathrm{m}^{2}$, once a day, d1 -3 , cytarabine $10 \mathrm{mg} / \mathrm{m}^{2}$, every 12 hours, d1-14, granulocyte colony stimulating factor $300 \mu \mathrm{g}$, once a day, d1-14, white blood cell count $>20 \times 10^{9} / \mathrm{L}$ when deactivated.

\subsection{Treatment of adverse events occurred during and principles}

According to $\mathrm{WHO}$, acute and subacute adverse drug reactions grading criteria during chemotherapy adverse reactions were graded evaluation. Before treatment, review of blood, liver function, renal function, periodic testing during treatment, suppression of bone marrow during chemotherapy for taking blood component transfusion, granulocyte colony stimulating factor, thrombopoietin methods such as supportive care, patients infected antibiotic therapy.

\subsection{Evaluation criteria}

Evaluation Criteria Evaluation Criteria reference IWG MDS International Working Group evaluated. At the end of each course of chemotherapy review of blood, blood rise when underwent bone marrow biopsy to assess the efficacy. 


\subsection{Follow-up}

All patients were followed until December 1, 2015. Mainly through telephone communication of patient follow-up. Follow-up data from the medical records. Cases of death during follow-up based on medical records or family members of patients with confirmed phone. Overall survival (OS) time was diagnosed with MDS to the time of death or clinical diagnosis of MDS to December 1, 2015.

\subsection{Statistical}

Description of the measurement data is represented by the median and range; statistical analysis with SPSS18.0 software. Effectiveness between the two groups were compared using $\chi^{2}$ test, agranulocytosis time and supportive treatment were compared using $\mathrm{t}$ test, $\chi 2$ test was used to compare the incidence of infection and bleeding incidence, Kaplan-Meier method to estimate time OS, OS rates between the two groups Log-rank test was used to compare.

\section{Results}

\subsection{Efficacy}

Observation of 31 cases of patients after one course of treatment clinically effective number of cases to 27 cases $(87.1 \%)$ achieved complete remission (CR) 22 cases $(71.0 \%)$, partial remission (PR) 5 cases (16.1\%), completely ineffective (hematology and bone marrow no improvement) 4 cases $(12.9 \%)$, with a median 1.7 courses of treatment, an average of 1.9 courses, the median OS time of 18.4 (6-56) months, an average of 28.6 months.

The control group of 37 patients with treatment after a course of treatment clinically effective number of cases to 26 cases $(70.3 \%)$ achieved complete remission (CR) 19 cases $(51.4 \%)$, partial remission (PR) 7 patients (18.9\%), completely ineffective ( hematology and bone marrow no improvement) in 11 patients $(29.7 \%)$, with a median 1.8 courses of treatment, an average of 2.3 months of treatment, the median time OS 10.6 (4-30) months, an average of 18.4 months (Table 1).

Table 1. The efficacy and survival of the two groups of patients with myelodysplastic syndrome.

\begin{tabular}{lllllll}
\hline \multirow{2}{*}{ groups } & \multirow{2}{*}{$\begin{array}{l}\text { Number } \\
\text { of cases }\end{array}$} & \multicolumn{2}{l}{ Efficacy (Example) } & \multirow{2}{*}{ ORR } & \multirow{2}{*}{ OS Time } \\
\cline { 3 - 5 } & CR & PR & R & & $18.4(6-56)$ \\
DAC+ half the Amount & 31 & 22 & 5 & 4 & 89.4 & $10.6(4-30)$ \\
of CAG & 37 & 19 & 7 & 11 & 80.1 & \\
\hline
\end{tabular}

Observation group and control group response rate was higher, $P<0.05$, the difference was statistically significant, overall survival was observed in the control group leader, the difference was statistically significant.

\subsection{Adverse reactions observed}

Adverse reactions occur after suppression of the bone marrow caused by infection, bleeding, selected 68 cases during treatment were inhibited bone marrow, the hematologic adverse reactions of grade 3-4 neutropenia average time of 13.3 days, on average the amount of red blood cells and blood cell transfusion need supportive treatment is 8 and $80 \mathrm{U}$, enrolled patients with varying degrees of infection, including 42 cases of infection 3-4, 1-2 grade infection in 12 cases, mainly for lung infection (56.3\%), followed by perianal infection $(29.3 \%)$, there are some patients with skin and soft tissue infections, urinary tract infection, 22 patients with varying degrees of gastrointestinal bleeding in some patients skin, mucous membranes, bleeding gums and other parts. All patients after anti-infection, bleeding, 
blood component transfusion and other symptomatic and supportive treatment, were safely through bone marrow suppression, see Table 2.

Table 2. Two adverse reactions in patients with myelodysplastic syndrome conditions and supportive care compared to the situation.

\begin{tabular}{lllllll}
\hline & & \multicolumn{2}{l}{ Adverse reactions } & \multicolumn{3}{l}{ Supportive care } \\
\cline { 3 - 7 } groups & $\begin{array}{l}\text { Number } \\
\text { cases }\end{array}$ & $\begin{array}{l}\text { The average } \\
\text { time of } \\
\text { agranulocyt } \\
\text { osis }(\mathrm{d} \pm \mathrm{s})\end{array}$ & $\begin{array}{l}\text { The } \\
\text { incidence of } \\
\text { infection }(\%\end{array}$ & $\begin{array}{l}\text { The } \\
\text { incidence of } \\
\text { bleeding }(\%)\end{array}$ & $\begin{array}{l}\text { RBC } \\
\text { transfusions } \\
(\mathrm{U} \pm \mathrm{s})\end{array}$ & $\begin{array}{l}\text { Platelet } \\
\text { transfusions } \\
(\mathrm{U} \pm \mathrm{s})\end{array}$ \\
\hline $\begin{array}{l}\text { DAC+ half } \\
\text { the Amount } \\
\text { of CAG }\end{array}$ & 31 & $14.4 \pm 1.8$ & 78 & 31 & $7.5 \pm 1.9$ & $78 \pm 14$ \\
CAG & 37 & $13.2 \pm 2.4$ & 81 & 33 & $7.6 \pm 1.4$ & $72 \pm 18$ \\
\hline
\end{tabular}

Lack of observed group and control group the mean granulocyte time difference was not statistically significant $(\mathrm{P}>0.05)$, two groups of patients the incidence of infection and the incidence of bleeding was no significant difference ( $>>0.05)$, two groups of red blood cells transfusions of platelets and the difference was not statistically significant $(\mathrm{P}>0.05)$.

Remission rate was significantly higher in patients in the observation group, adverse reactions and therapeutic support there was no difference.

\section{Discussion}

In this study were selected two groups of patients with MDS IPSS risk groups for the intermediate- or high-risk patients, patients with a high risk of this type of transformation to leukemia [1-2]. Because such patients, mainly in the elderly, although it still can not give a positive chemotherapy get a higher response rate and longer lifetime. In particular, the conventional CAG program, due to cardiac toxicity of anthracyclines and cytotoxic chemotherapy drugs, often resulting in older patients can not tolerate. With the gradual deepening of epigenetic research, we found that in the process of hematopoiesis high-risk MDS patients, some start factor methylation occurred, resulting in some important gene silencing, inactivated, thereby causing disease gradually progress to leukemic transformation. Bacher $\mathrm{U}$, Mihara $\mathrm{k}$ [3-4] and put forward a higher proportion of P15 gene MDS patients in cell cycle regulation (INK4b) abnormal methylation. On the basis of these theoretical studies, in 2006, decitabine as a specific DNA methylation inhibitor approved by the US FDA listed, Zagonel V [5], etc. The study shows that decitabine effective treatment of MDS and thus widely used in clinical, but dose decitabine did not reach a consensus. Thereafter through a lot of studies have found that high concentrations of decitabine main play its cytotoxicity, while low concentrations allows DNA methyltransferase enzyme inactivation does not lead to cell death [6]. Many scholars through a lot of tests to explore the application of decitabine dose, while exploring single land decitabine MDS effect is more ideal. Finally, Chowdhury S [7] found that application of low-dose decitabine with gemtuzumab combined treatment of relapsed or refractory acute myeloid leukemia achieved better results. Since then many researchers have studies with decitabine in combination can improve the efficacy of the program MDS. Qin [8] study found that decitabine in inhibiting DNA demethylation, while also improving the cytotoxicity of cytarabine, G-CSF or GM-CSF can increase the sensitivity of cell cycle dependent drug so in theory decitabine for MDS patients with joint CAG program should be a good effect. Qian [9] reported that patients with high-risk MDS applications to decitabine joint CAG regimen, complete remission rate reached $82.6 \%$. High $\mathrm{Su}$ [10] pointed out that decitabine monotherapy and combination regimens half and full CAG myelodysplastic syndrome and acute myeloid leukemia have a good effect; Zhang Pingyun [11] found that a small dose of diazepam Combined CAG gemcitabine chemotherapy in high-risk patients with myelodysplastic syndrome more effective than chemotherapy alone in patients with CAG. This study showed that decitabine Joint 
CAG half the amount in high-risk myelodysplastic syndrome exception has a good effect, this result is consistent with the HL group [12] and other findings alone with no difference in efficacy of CAG regimen; in survival time aspect, decitabine joint leader in half the amount of CAG CAG regimen alone group, but the difference was not statistically significant, given the small sample size related. But we should pay attention to, such as the corresponding incidence of infection, bleeding and other complications are also higher incidence of adverse reactions and supportive care aspects of the research groups studied, decitabine joint half dose group and CAG CAG regimen alone no difference in the treatment group; Steensma [13] and so noted in the ADOPT trial, patients with pulmonary infection probability $11 \%$ probability of pulmonary infection in this study is much higher than this result, consider the patient to recognize laminar flow ward inadequate, poor economic conditions, more and more people in our hospital general wards, increasing the chance of cross-infection related. Recommended in the early chemotherapy, proper use of drugs to prevent fungal and anti-bacterial treatment to reduce the risk of infection may be to a certain extent, this view is consistent with the Jain [14] of.

\section{References}

1. Nimer SD. Myelodysplastic syndromes.Blood,2008;111(10):4841-4845.

2. Nolte F, Hofnann WK. Molecular mechanisms involved in the progression of myelodysplastic syndromes.Future Oncol,2010;6(3):445-455.

3. Bacher U, Haferlach T, Kern W,et al. A comparative study of molecular mutations in 381 patients with myelodysplastic syndrome and in 4130 patients with acute myeloid leukemia. Haematologica, 2007;92(6):744-752.

4. Mihara K, Takihapa Y, Kimura A. Genetic and epigenetic alterations in myelodysplastic syndrome. Cytogenet Cenome Res,2007;118(2-4):297-303.

5. Zagonel V, Lo Re G, Marotta G,et al. 5-Aza-2' -deoxycytidine(Decitabine) induces trilineage reponse in unfavourable myelodysplastic syndromes. Lukemia,1993;7(suppl 1):30-35.

6. OKI Y, AOKI E, ISSA J P, et al. Decitabine-Bedside to bench[J].Crit Rev Oncol Hematol,2007, 61(2):140-152.

7. Chowdhury S, Seropian S, Marks PW. Decitabine combined with fractionated gemtuzumab ozogamicin therapy in patients with relapsed or refractory acute myeloid leukemia[J].Am $J$ Hematol, 2009,84(9):599-600.

8. Qin T, Youssef EM, Jelinek J,et al. Effect of cytarabine and decitabine in combination in human leukemic cell lines[J].Clin Cancer Res,2007,13(14):4225-4232.

9. Qian S, Duan L. Effect of low-dose cytarabine and aclarubicin in combination with decitabine on the outcome of patients with high-risk myelodysplastic syndromes[G].12th International Symposium on Myelodyspl- astic Syndromes.Berlin, Germany: Leukemia Research,2013:S152S153.

10. The high $\mathrm{Su}$, Chou Huiying, Jin Zhengming etc. decitabine monotherapy and combination programs CAG half and full observation [J] Efficacy in treating abnormal myelodysplastic syndrome, leukemia and acute myeloid. Journal of Hematology 2014,35 (11):961-964 (Chin J Hematol, November2014, Vol.35, No.11)

11. Tibet, Wu Wenzhong, Cui Guoxing, decitabine and other programs and joint CAG CAG regimen alone, and compare the efficacy of high-risk myelodysplastic syndrome [J] Chinese Journal of Experimental Hematology, (Journal of Experimental Hematology) 2014;.. 22 (5) :. 1341-1344

12. HL group, Dengyin Fen, Zhang Lei, et decitabine sequential half the amount of CAG regimen in high-risk myelodysplastic syndrome and leukemia transformation of four cases [J], leukemia. Lymphoma 2012; 21 (7);. 433-435 (Journal of Leukemia \& Lymphoma, July2012, Vol, 21, No.7)

13. Steensma DP, Baer MR, Slack JL, et al. Multicenter study of decitabine administered daily for 5 days every 4 weeks to adults with myelodysplastic syndromes: the alternative dosing for outpatient treatment(ADOPT)trial [J].J Clin Oncol,2009,27(23):3842-3848. 
14. Jain N, Mattiuzzi GN, Cortes J, et al. Benefit of anti-infectious prophylaxis in patients with acute myeloid leukemia or high-risk myelodysplastic syndrome receiving frontline"targeted therapy"[G].49th American Society of Hematology Annual Meeting. Atlanta, Georgia: Blood,2007:2858. 